\title{
Eksenel fonksiyonel derecelendirilmiş helislerin karışık sonlu eleman yöntemi ile serbest titreșim analizi
}

\author{
Free vibration analysis of axially functionally graded helices via mixed finite \\ element method
}

\author{
Merve Ermiş ${ }^{1, *}$ \\ ${ }^{1}$ Kırklareli Üniversitesi, İnşaat Mühendisliği Bölümü, 39060, Kayalı Kampüsü Merkez, Kırklareli Türkiye
}

\begin{abstract}
Özet
Bu çalışmanın amacı, eksenel fonksiyonel derecelendirilmiş (EFD) kesin geometri tarifi üzerinden elde edilen dairesel olmayan (fiçl, hiperboloidal ve eliptik) helislerin doğal frekanslarını incelemektir. Timoshenko cubuk kuramı üstünden geliştirilen karışık sonlu eleman yönteminde kesit çarpılması da gözetilerek serbest titreşim analizi yapılmıştır. İki düğüm noktalı eğrisel sonlu elemanın her düğüm noktasındaki 12 değișken; üçü yer değiştirmeler, üçü kesit dönmeleri, üçü kuvvetler ikisi eğilme biri burulma momentleridir. Eksenel FD dairesel olmayan geometriye sahip ve kesin geometri üzerinden tariflenen helislerin serbest titreşim analizi farkl sınır koşullarl ve malzeme gradyenti değişimleri üzerinden detaylıca tartısılmıstır. Yöntem literatür ya da ANSYS ile doğrulandıktan sonra literatür için tamamen özgün problemler çözülmüştür.
\end{abstract}

Anahtar kelimeler: Kesin helis geometrisi, Fonksiyonel derecelendirilmiş malzeme, Sonlu eleman, Çarpılma, Serbest titreşim analizi

\section{Giriş}

Fonksiyonel derecelendirilmiş malzemeler, hafif, yüksek mukavemetli, ısıl direnç özellikli, aşınmaya veya korozyona karşı dayanımlı olmaları nedeni ile uzay, havacılık, endüstriyel malzemeler, biyoloji, enerji ve savunma sanayiinde kullanılmaktadır [1]. Bunlara örnek olarak, otomotiv turboşarjlar, termal bariyer kaplamalar, katı oksit yakıt hücreleri ve biyomedikal aletler verilebilir [2-6]. Ayrıca helisel geometri, nano ölçekten makro ölçeğe kadar mühendislik tasarımında yaygın bir kullanım alanına sahiptir. Bunlara örnek olarak, helisel karbon nano-tüpler, helisel polimerler, helisel erişim düzenekleri, biyomedikal aletler, mekanik yaylar, merdivenler, yapı titreşimini azaltmada kullanılan helisel izolatörler verilebilir [7-13].

Bir kirişte eksenel fonksiyonel derecelendirilmiş (EFD) malzeme, kiriş ekseni boyunca sürekli değişen malzeme özellikleri ile karakterize edilir. Literatür incelendiğinde, eksenel FD kirişler/nano-kirişler ile ilgili çalışmaların çoğu doğru eksenli çubuklar üzerinden yürütülmüştür, bu çalışmalardan bazıları [14-30] dır. Eksenel FD doğru eksenli çubuk çalışmalarına kıyasla, eksenel FD düzlem eğrisel çubuklar ile ilgili çalışmaların sayısı daha azdır ve bu çalışmalardan bazıları: Rajesekaran [31], eksenel FD değişken kesitli eğrisel çubukların statik ve serbest titreşim analizini kayma ve dönel eylemsizlik etkilerini dahil ederek incelemiştir. Tsiatas ve Charalampakis [32], eksenel FD kiriş ve dairesel düzlem çubukların doğal frekanslarının

\section{Abstract}

The objective of this study is to investigate the natural frequencies of axially functionally graded (AFG) non-circular (barrel, hyperboloidal and elliptical) helices based on exact geometry. Free vibration analysis is performed using the mixed finite element method based on Timoshenko beam theory by considering cross-sectional warping effect. A two-noded curved finite element involves 12 field variables at each node, three displacements, three cross-sectional rotations, three forces, and three moments. The free vibration analysis of axially $F G$ exact non-circular helical geometries is discussed in detail over different boundary conditions and material gradient indexes. After verifying the algorithm with the problems available in the literature and ANSYS software, original problems for the literature are solved.

Keywords: Exact helix geometry, Functionally graded material, Finite element, Warping, Free vibration analysis

optimizasyonu problemi üzerinde çalışmışlardır. Lee ve Lee [33], deneme (trial) özdeğer yöntemi ile geliştirilmiş doğrudan integral yöntemi kullanılarak eksenel FD dairesel kirişlerin serbest titreşim davranışını incelemişlerdir. Noori vd. [34], eksenel FD parabolik çubukların hem serbest titreşim hem de zorlanmış titreşim problemini tamamlayıcı fonksiyonlar yöntemini kullanarak çözmüşlerdir. Temel ve Noori [35], değişken kesite sahip eksenel FD sikloit çubukların düzlem dışı titreşim davranışını [34] de sunulan yöntem ile incelemişlerdir. Literatürde dairesel olmayan eksenel FD üç boyutlu helisel çubuklar ile ilgili yalnızca bir adet yayına rastlanmıştır. Bu yayında, Calim ve Cuma [36] eksenel FD değişken kesite sahip fiçı ve hiperboloidal helislerin serbest titreşim analizi problemini transfer matris ve rijitlik matris yöntemlerini kullanarak yaklaşık helis geometrisi tarifi üzerinden incelemişlerdir.

Yapısal analizlerle ilgili çalışmalar göz önüne alındığında, yaklaşık geometriye dayalı dairesel olmayan helislerin dinamik davranışlarını inceleyen çok sayıda çalışma mevcuttur. Bunların çoğu serbest titreşim analizini [37-44], ve birkaçı $[45,46]$ ise zorlanmış titreşim analizi ile ilgilidir. Dairesel olmayan bir helis geometrisi ekseni boyunca değişen eğrilik ve tabii burulmaya sahiptir. Genel (Lancret) helis tanımına göre, eksen boyunca eğrilik ve tabii burulma arasindaki oran sabittir [47, 48]. Dairesel helisin kesin geometrik fonksiyonları (helisin eğriliği, tabi burulması, yay boyu gradyenti, yükselme açısı ve yükseklik)

\footnotetext{
* Sorumlu yazar / Corresponding author, e-posta / e-mail: mermis@klu.edu.tr (M. Ermis)

Geliş / Recieved: 09.11.2020 Kabul / Accepted: 16.12.2020 Yayımlanma/Published: 15.01.2021

doi: 10.28948/ngmuh.823385
} 
dejenere edilerek dairesel olmayan helislerin yaklaşık fonksiyonları elde edilmektedir [38]. Ermis ve Omurtag [49], Lancret türü dairesel olmayan helislerin geometrik fonksiyonlarını kesin ve yaklaşık geometri tarifi üzerinden sunmuşlardır. Daha sonra çalışmalarında, yaklaşık helis geometrisi tanımının sınırlı olduğu aralığı düzlem eğrisi Archimedean veya logaritmik spiralden oluşan konik helisler için parametrik bir çalışma ile kesin ve yaklaşık fonksiyon (helisin eğriliği, tabi burulması, yay boyu gradyenti) sonuçları üzerinden ortaya koymuşlardır. Çalışmalarının yapısal analiz ile ilgili kısmında ise, karışık sonlu eleman ve yer değiştirme türü sonlu eleman yöntemlerinin performanslarını kesin geometri tarifi üzerinden tanımlanan ve Archimedean spiralden oluşan konik helisin statik ve serbest titreşim analizi üzerinden detaylı olarak incelemişlerdir. Ermis [50], Archimedean spiralden oluşan konik helis için [49] da sunulan yaklaşık geometrinin sınırlı olduğu aralıkta, yapısal analiz sonuçlarının bu dejenerasyon işleminden hangi oranlarda etkilendiği problemini statik ve serbest titreşim analizleri üzerinden ele almıştır. Helisel çubukların yapısal analiz sonuçlarına ait hassasiyet hem eksen geometrisinin kesin ifadesine hem de dairesel olmayan kesitlerdeki çarpılma etkisinin dahil edilip edilmemesine bağlıdır. Araştırmalar, eğri eksenli çubuklara uygulanan dış yüklerin önemli mertebelerde bir burulma etkisi yaratma eğiliminde olduğunu göstermektedir ve bu yüzden kesit çarpılmasının gözetildiği burulma rijitliğinin hassas olarak hesaplanması hususunda ayrı bir çaba harcanmaktadır [5153]. Aribas [54], karışık sonlu eleman analizi ile kompozit eğrisel kirişlerin kesit çarpılması gözetilen normal ve kayma gerilmelerini incelemiştir. Aribas vd. [55], kesitteki çarpılma etkisini kompozit tabakalı ve kesin geometri tarifi üzerinden tanımlanan konik helislerin statik ve serbest titreşim analizi üzerinden karışık sonlu elemanlar formülasyon yöntemi ile detaylı olarak araştırmışlardır. Arıbas vd. [56], kesitteki çarpılma etkisinin dahil edildiği normal ve kayma gerilmelerini, tabakalı kompozit- geometrik olarak kesin eliptik konik helislerin zorlanmış titreşim analizi üzerinden karışık sonlu elemanlar yöntemiyle incelemişlerdir. Jog ve Mokashi [51] tarafından sunulan yer değiştirme bazlı sonlu elemanlar kullanılarak kesit çarpılması gözetilen burulma rijitliği Arıbas [54], Arıbas vd. [55, 56] da iki düğüm noktalı eğrisel sonlu eleman matrisine eklenmiştir. Dairesel olmayan kesite sahip yapıların lineer olmayan ve burkulma analizleri üzerinde çarpılmanın etkisi oldukça önemlidir [57-60].

Lancret tipi dairesel olmayan helislerin [49] da verilen kesin geometri tanımı, [49] da düzlem eğrisi Archimedean veya logaritmik spiralden konik helisler, [50] de fiçı helis ve [56] da ise eliptik ve eliptik konik helisler için sunulurken; bu çalışma kapsamında hiperboloidal helise uygulanmıştır. Daha sonra, bu çalıșma ile Timoshenko çubuk kuramı üzerinden eksenel FD dairesel olmayan fiçı, hiperboloidal ve eliptik türü helisel çubukların serbest titreşim analiz problemini ele alan karıșık sonlu eleman formülasyonu, [5456] da verilen çarpılma etkisinin de dâhil edildiği durum gözetilerek sonlu eleman matrisleri elde edilmiştir. Kesitteki çarpılmanın yanı sıra kayma etkileri ve dönel eylemsizlikler de dikkate alınmıştır. Parametrik çalışmalarda, farklı sınır koşullarının, malzeme gradyenti değerlerinin ve dairesel olmayan helis geometrilerinin etkileri incelenmiştir. $\mathrm{Bu}$ çalışmada kesin geometri tarifi üzerinden ele alınan eksenel FD helisel çubuk örneklerinin tümü literatür için orijinaldir ve bazı kritik örnekler ise sonlu eleman yazılımı olan ANSYS'in SOLID186 [61] elemanlarından elde edilen sonuçlarla kıyaslanarak doğrulanmıştır. Yazarın bilgisi dahilinde, kesin geometri tarifi üzerinden eksenel FD dairesel olmayan Lancret tipi helislerin serbest titreşim analizi problemi literatürde ilk defa bu çalışmada ele alınmıştır.

\section{Materyal ve metot}

$\mathrm{Bu}$ bölümde kesin helis geometrisi, helisel çubuk geometrisine ait alan denklemleri ve fonksiyonel, karışık sonlu eleman formülasyonu ile eksenel FD malzeme formülasyonu verilmiştir.

\subsection{Kesin helis geometrisi}

Sirasiyla, $s$ ve $\varphi$ genel helisin yay boyu ve yatay açısının göstermek üzere, helise ait kesin geometrik fonksiyonlar şu şekilde sıralanabilir: $\mathbf{r}^{p}(\varphi)=\{x(\varphi), y(\varphi), z(\varphi)\} \quad$ helisin $\quad x-y \quad$ düzleminde bulunan ilgili düzlem eğrisini göstermektedir. Helisin eğriliği $\chi(\varphi)=\chi^{p}(\varphi) \cos ^{2}(\alpha) \quad$ ve tabii burulması $\tau(\varphi)=\chi^{p}(\varphi) \cos (\alpha) \sin (\alpha)$, ilgili düzlem eğrinin eğriliği $\chi^{p}(\varphi)=\left\|\mathbf{r}_{, s s}^{p}\right\|$ kullanılarak elde edilir. $\alpha=90^{\circ}-\theta$ helisin yükselme açısıdır, $\theta$ ise helis açısıdır (Bkz. Şekil 1). Üst indis $p$ düzlem eğriyi ifade etmektedir ve $\mathbf{r}^{p}$ nin alt indisindeki virgül ise $s$ parametresine göre türevi göstermektedir. Helisin diferansiyel yay boyu $\mathrm{d} s=c(\varphi) \mathrm{d} \varphi$ ile tanımlanır burada $c(\varphi)=\left\|\mathbf{R}_{, \varphi}\right\|$ yay boyunun gradyentini göstermektedir. $\mathbf{R}(\varphi)$, Kartezyen koordinat sisteminde genel helislerin yer vektörünü tanımlamaktadır (Bkz. Şekil 1), global eksende düşey koordinat $z=\tan (\alpha) \int_{0}^{\varphi}\left\|\mathbf{r}_{, \psi}^{p}(\psi)\right\| \mathrm{d} \psi$ eşitliği yardımıyla elde edilir.

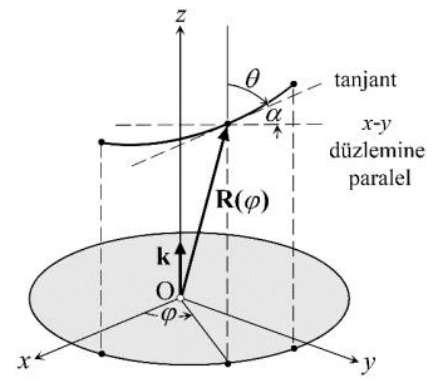

Şekil 1. Genel helis parçası. $\theta$ : helis açısı, $\alpha$ : yükselme açısı, $\varphi$ : yatay açı, $\mathbf{k}$ : Darboux gösterici

Frenet birim vektörleri $\mathbf{t}, \mathbf{n}, \mathbf{b}$ sirasıyla teğet, normal ve binormal birim vektörlerdir. Yukarıda bahsedilen fonksiyonlar, yükseklik/yükselme açısı ve Kartezyen koordinatlar ile Frenet koordinatları arasındaki dönüşüm 
Tablo 1. Dairesel olmayan helislerin $x-y$ bileşenleri. $0 \leq \varphi \leq 2 \pi n$ burada $n$ tur sayısıdır.

\begin{tabular}{ccc}
\hline Helis Tipi & $x(\varphi)$ & $y(\varphi)$ \\
\hline Fiçı & {$\left[R_{\text {max }}+\left(R_{\min }-R_{\text {max }}\right)(1-(\varphi / \pi n))^{2}\right] \cos \varphi$} & {$\left[R_{\max }+\left(R_{\min }-R_{\max }\right)(1-(\varphi / \pi n))^{2}\right] \sin \varphi$} \\
Hiperboloidal & {$\left[R_{\min }+\left(R_{\max }-R_{\min }\right)(1-(\varphi / \pi n))^{2}\right] \cos \varphi$} & {$\left[R_{\min }+\left(R_{\max }-R_{\min }\right)(1-(\varphi / \pi n))^{2}\right] \sin \varphi$} \\
Eliptik & $R_{\max } \cos \varphi$ & $R_{\min } \sin \varphi$ \\
\hline
\end{tabular}

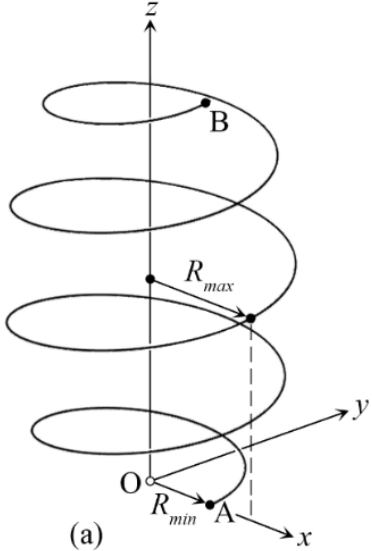

(a)
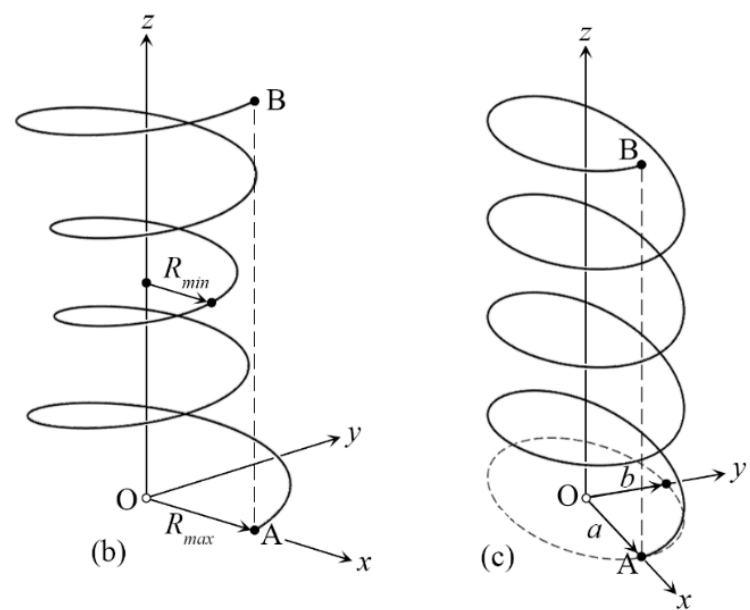

Şekil 2. Dairesel olmayan kesin helis geometrileri (a) fiçı helis, (b) hiperboloidal helis ve (c) eliptik helis ( $a=R_{\max }$,

$\left.b=R_{\min }\right)$

matrisi ile ilgili detaylı bilgi Ermis ve Omurtag [49] da mevcuttur. Fıçı, hiperboloidal ve eliptik helislere ait ilgili düzlem eğrilerinin $\mathbf{r}^{p}(\varphi) \quad x y$ düzlemindeki bileşenleri Tablo 1'de verilmiştir. Burada $R_{\max }$ ve $R_{\min }$ (Bkz. Şekil 2) sırasıyla en büyük ve en kü̧̧ük yarıçap değerlerini göstermek üzere, tüm dairesel olmayan helis türleri için $\xi=R_{\min } / R_{\max }$ oranın tanım aralığı $0<\xi<1$ dir.

\subsection{Fonksiyonel ve karışılk sonlu eleman formülasyonu}

Homojen izotrop helisel çubukların serbest titreşim ve statik analizine ait fonksiyonel [44, 62] verilmiştir. Karışık sonlu elaman formülasyonunda kullanılan fonksiyonel, Gâteaux türevi ve [63] de verilen potansiyel operatör konsepti kullanılarak elde edilmiştir. Bu çalışmada, Jog ve Mokashi [51] tarafından sunulan yer değiştirme bazlı sonlu elemanlar kullanılarak kesit çarpılması gözetilen burulma rijitliği [54-56] çalıșmalarında sunulduğu gibi iki düğüm noktalı eğrisel sonlu eleman matrisine eklenmiştir ve daha önceki çalışmalarda izotrop ve homojen malzemeden oluşan helisel çubuğa ait fonksiyonel ve alan denklemleri, bu çalışma kapsamında eksenel FD helisel çubukların serbest titreşim analiz problemini ele alacak biçimde genişletilmiştir. Eksenel FD helislerin serbest titreşimi için elde edilen fonksiyonel aşağıda verilen Denklem (1)'deki gibi elde edilir.

$\mathbf{u}\left(u_{t}, u_{n}, u_{b}\right)$ yer değiş̧irme vektörünü, $\Omega\left(\Omega_{t}, \Omega_{n}, \Omega_{b}\right)$ kesit dönmesi vektörünü, $\quad \mathbf{T}\left(T_{t}, T_{n}, T_{b}\right) \quad$ kuvvetleri ve $\mathbf{M}\left(M_{t}, M_{n}, M_{b}\right)$ moment vektörünü, $\rho=\rho(s)$ malzeme yoğunluğunu, $A$ kesit alanını, I eylemsizlik moment vektörünü, $\mathbf{C}_{\gamma}$ ve $\mathbf{C}_{\kappa}$ kompliyans matrislerini, $\omega$ ise doğal açısal frekansı göstermektedir.

$$
\left.\begin{array}{rl}
\mathbf{I}(\mathbf{y})= & -\left[\mathbf{u}, \mathbf{T}_{, s}\right]+[\mathbf{t} \times \boldsymbol{\Omega}, \mathbf{T}]-\left[\mathbf{M}_{, s}, \boldsymbol{\Omega}\right]-\frac{1}{2}\left[\mathbf{C}_{\kappa} \mathbf{M}, \mathbf{M}\right] \\
& -\frac{1}{2}\left[\mathbf{C}_{\gamma} \mathbf{T}, \mathbf{T}\right]-\frac{1}{2} A \omega^{2}[\rho \mathbf{u}, \mathbf{u}]-\frac{1}{2} \omega^{2}[\rho \mathbf{I} \boldsymbol{\Omega}, \boldsymbol{\Omega}] \\
& +[(\mathbf{T}-\hat{\mathbf{T}}), \mathbf{u}]_{\sigma}+[(\mathbf{M}-\hat{\mathbf{M}}), \boldsymbol{\Omega}]_{\sigma}+[\hat{\mathbf{u}}, \mathbf{T}]_{\varepsilon}+[\boldsymbol{\Omega}, \mathbf{M}]_{\varepsilon}
\end{array}\right\}
$$

Denklem (1)'de verilen fonksiyoneldeki köşeli parantezler iç çarpımları ifade etmekte olup, şapkalı terimler sınırlardaki bilinen değerleri ifade etmektedir ve alt indis $\varepsilon$ ve $\sigma$ ise sırasıyla geometrik ve dinamik sınır koşullarını göstermektedir. İki düğüm noktalı eğrisel eleman her düğüm noktasında 12 tane değişkene sahiptir, Frenet koordinat takımında tanımlanan bu değişkenlerden üçü yer değiştirmeleri, üçü kesit dönmelerini, üçü kuvvetleri ve diğer üçü ise momentleri göstermektedir. Bu çalışmada ele alının fiçı, hiperboloidal ve eliptik helislerin eleman rijitlik ve kütle matrislerinin analitik fonksiyonları kesin geometri tarifi üzerinden tanımlanan eğrilik, tabii burulması ve yay gradyenti üzerinden Ermis ve Omurtag [49] da belirtilen işlem adımları takip edilerek oluşturulmuştur. Dairesel olmayan kesitlerde çarpılmanın dahil edildiği burulma rijitliği eleman matrisine entegre edilmiştir. 


\subsection{Eksenel fonksiyonel derecelendirilmiş malzeme}

Eksenel FD malzemenin dağılımı üssel fonksiyon olarak değiştiği kabul edilmiştir. Boyu $L$ olan doğru eksenli çubuklar için Denklem (2):

$$
g(x)=g_{0}+\left(g_{1}-g_{0}\right)\left(\frac{x}{L}\right)^{m}, \quad 0 \leq x \leq L
$$

Tur sayısı $n$ olan helisel çubuklar için Denklem (3):

$$
g(\varphi)=g_{0}+\left(g_{1}-g_{0}\right)\left(\frac{\varphi}{2 \pi n}\right)^{m}, \quad 0 \leq \varphi \leq 2 \pi n
$$

Burada, $g$ malzeme özelliklerinden elastisite modülünü ( $E$ ) ve malzeme yoğunluğunu $(\rho)$ göstermektedir, $m$ malzeme gradyenti parametresini, alt indis "0" ve "1" ise çubuk ekseni boyunca $x$ ve $\varphi$ değerlerinin ifade ettiği başlangiç $(x=0, \varphi=0)$ ve bitiş $(x=L, \varphi=2 \pi n)$ noktalarındaki malzemeleri göstermektedir (Bkz. Şekil 3, Denklemler (2)-(3)). Burada, $x$ doğru eksenli çubuk eksenini ve $\varphi$ ise helisin $x y$ düzlemindeki yatay açısını temsil etmektedir (Şekil 1). Malzeme sabitleri $E_{0}=200 \mathrm{GPa}, \quad \rho_{0}=5700 \mathrm{~kg} / \mathrm{m}^{3}, \quad E_{1}=70 \mathrm{GPa}$, $\rho_{1}=2702 \mathrm{~kg} / \mathrm{m}^{3}$ ve Poisson oran $v=0.3$ olarak seçilmiştir. Denklem (3) de helisel çubuklar için verilen eksenel FD malzeme özelliğinin değişim fonksiyonu, farklı malzeme gradyent parametreleri $m=0.5,1,2,3$ ve tur sayıs $n=4$ için yatay açı $\varphi$ ye göre değişimi çizilmiştir (Bkz. Şekil 3). Şekil 4'te ise eksenel FD fıçı helis örneği verilmiştir.

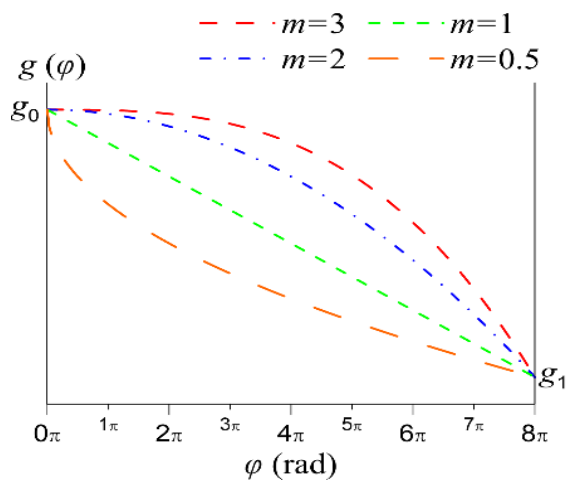

Şekil 3. Malzeme özellikleri fonksiyonu $g(\varphi) \operatorname{nin}$ $0 \leq \varphi \leq 2 \pi n(n=4)$ aralığında farklı malzeme gradyent parametre değerlerine $m=0.5,1,2,3$ göre değişimi.

\section{Bulgular ve tartışma}

\subsection{Karışık SEY eksenel FD doğru eksenli çubuk için literatür ile kıyaslanması}

Eksenel FD malzemenin karışık sonlu SEY ile çözümü literatürde ([19, 22]) bulunan eksenel FD doğru eksenli çubuğa ait açısal frekans değerleri üzerinden doğrulanmıştır.
Malzeme parametreleri ve malzemenin çubuk ekseni boyunca değişimine ait fonksiyon bölüm 2.3 de verilmiştir, malzeme gradyent parametresi $m=1,2$ ve 3 seçilmiştir. Birimsiz atalet yarıçapı $r=I /\left(A L^{2}\right)=0.01$ ve birimsiz

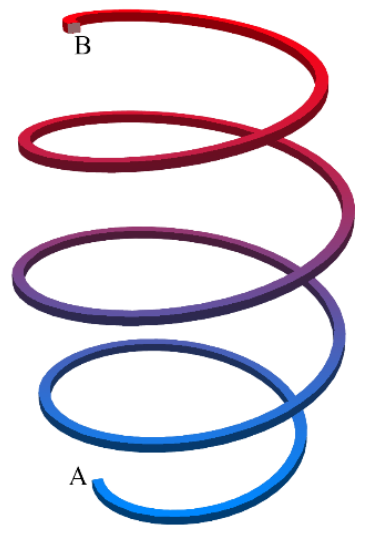

Şekil 4. Eksenel FD fiçı helis örneği

doğal frekans parametresi ise $\Gamma=\omega \sqrt{\left(\rho_{0} A L^{4}\right) /\left(E_{0} I\right)}$ olup burada $A$ ve $I$ sirasıyla kesit alanını ve eylemsizlik momentini göstermektedir. $n_{e}=40$ sonlu eleman ait birinci doğal frekans sonuçları, $n_{e}=50$ sonlu elemana ait sonuçlar üzerinde normalize edildiğinde, Tablo 2 de verilen sınır koşulları (SK) ve malzeme gradyenti parametreleri için aradaki fark \%0.01 den azdır. Yakınsama analizi sonuçlarına göre, Tablo 2'de eksenel FD doğru eksenli çubuğun birimsiz doğal frekans parametresi $(\Gamma) n_{e}=50$ sonlu eleman sonuçlarına göre sunulmuştur. Eksenel FD doğru eksenli çubuğun karışık SEY (bu çalışma) ile elde edilen sonuçlar literatür sonuçları ile oldukça uyumlu olduğu gözlemlenmiştir.

Tablo 2. Eksenel FD doğru eksenli çubuğun birimsiz doğal frekans parametresi $\Gamma$.

\begin{tabular}{cccccc}
\hline SK & $m$ & {$[19]^{1}$} & {$[19]^{2}$} & {$[22]$} & $\begin{array}{c}\text { Bu } \\
\text { çalışma }\end{array}$ \\
\hline ankastre- & 1 & 3.8963 & 3.8963 & 3.896370 & 3.8962 \\
serbest & 2 & 3.8828 & 3.8828 & 3.882898 & 3.8828 \\
& 3 & 3.7957 & 3.7956 & - & 3.7958 \\
\hline sabit- & 1 & 7.8459 & 7.8459 & 7.846103 & 7.8456 \\
sabit & 2 & 7.9873 & 7.9872 & 7.987477 & 7.9867 \\
& 3 & 8.0645 & 8.0644 & - & 8.0636 \\
\hline \multirow{2}{*}{ ankastre- } & 1 & 12.9065 & 12.9064 & 12.90720 & 12.906 \\
ankastre & 2 & 12.6873 & 126871 & 12.68796 & 12.686 \\
& 3 & 12.6030 & 126028 & - & 12.600 \\
\hline
\end{tabular}

$\mathrm{DTEM}^{1}$ : Diferansiyel dönüşüm eleman yöntemi, $\mathrm{DQEL}^{2}$ :en düşük mertebeli diferansiyel kuadratik eleman

\subsection{Eksenel fonksiyonel derecelendirilmiş dairesel olmayan helisler}

$\mathrm{Bu}$ örnekte, farklı sınır koşullarına sahip ve kesin geometri üzerinden tanımlanan eksenel FD dairesel olmayan (fıçı, eliptik, hiperboloidal) helisel çubukların ilk beş doğal frekansı, farklı malzeme gradyent parametreleri üzerinden araştırılmıştır. Tüm dairesel olmayan helis geometrileri için 
ortak parametreler: helis yüksekliği $H=45 \mathrm{~mm}$, tur sayısı $n=4, \xi=R_{\min } / R_{\max }=0.6$ olup en büyük yarıçap değeri $R_{\max }=15 \mathrm{~mm}$ dir. Dikdörtgen kesit genişliği $w=1 \mathrm{~mm}$ olup yüksekliği ise $h=0.8 \mathrm{~mm}$ ( $b$ eksenine paralel) dir. Malzeme parametreleri ve helis ekseni boyunca değişimleri bölüm 2.3 de (Bkz. Şekil 3) verilmiştir.

\subsubsection{Yakınsama analizi}

İlk olarak, 3B eğrisel yapı sisteminde eksen boyunca fonksiyonel değişen malzeme parametreleri için yapının serbest titreşim davranışını yansıtan karışı sonlu eleman formülasyonun yakınsama analizi yapılacaktır. Bu analiz, malzeme değişim parametresi $m=1$ ve iki ucu tutulu sınır koşuluna sahip eksenel FD fiçı, eliptik ve hiperboloidal helislerin birinci doğal frekans değerleri üzerinden yapılmış olup Şekil 5 'te eleman sayısına $\left(n_{e}\right)$ göre çizilmiştir.
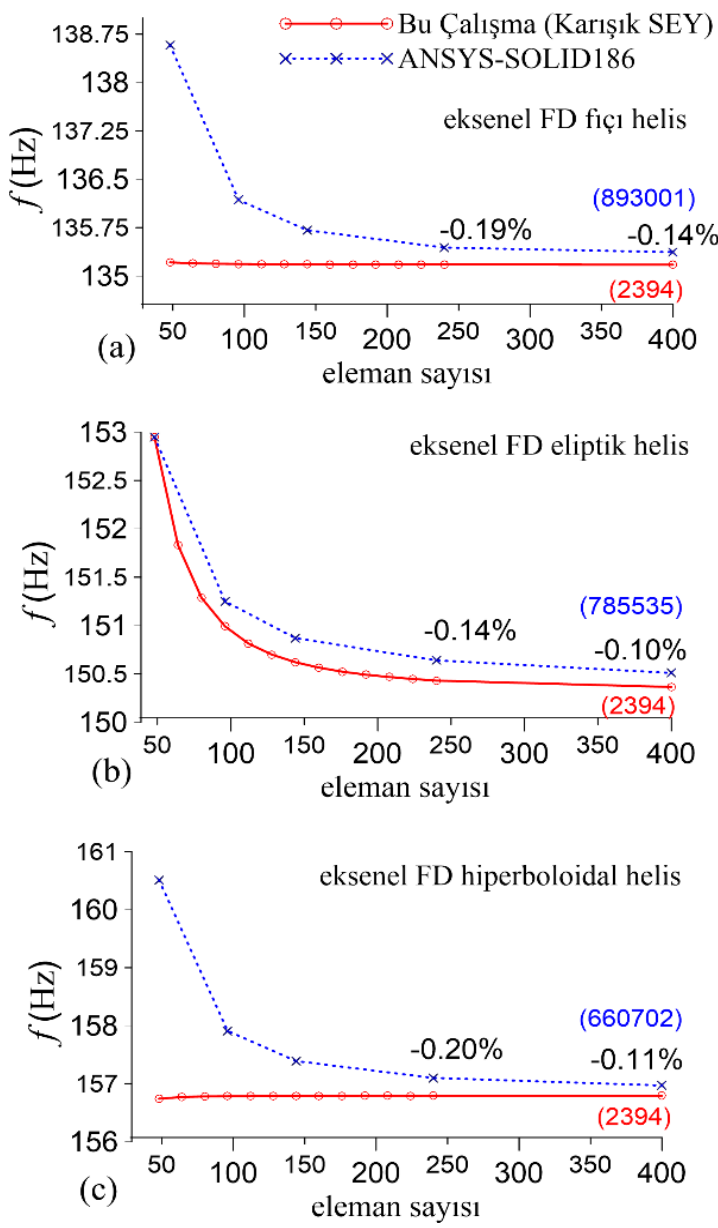

Şekil 5. Eksenel FD fıçı, eliptik ve hiperboloidal helislerin birinci doğal frekans değerleri $(f)$ için bu çalışma (karış1k SEY) ve ANSYS-SOLID186 çözümlerine ait yakınsama analizi

ANSYS'den elde edilen sonuçlar karısıık SEY'den elde edilen sonuçlara göre normalize edilerek yüzde farklar Şekil 5'te verilmiştir, ayrıca karışı SEY ve ANSYS-SOLID186 elemanına ait bilinmeyen sayıları parantez içinde sunulmuştur. ANSYS-SOLID186 elemanda, malzeme özellikleri iki boyutlu eğrisel elemanın (Bkz. Şekil 6) düğüm noktalarındaki malzeme değerlerinin ortalaması olarak tanımlanmıştır. Şekil 5’te, karıșı SE yöntemi kullanılarak hesaplanan fiçı ve hiperboloidal helisin birinci doğal frekans değerlerinin yakınsama hızının ANSYS-SOLID186 eleman kullanılarak elde edilen sonuçlara göre daha hızlı olduğu görülmektedir. Hatta, karışı sonlu eleman formülasyon sonuçları için frekans değerlerinin daha seyrek ağ sıklaştırma bölgelerinde bile yakınsadığı gözlenmektedir. Eliptik helise ait yakınsama hızı fiçı ve hiperboloidal helislere ait yakınsama hızları ile kıyaslandığında hem karışık SEY için hem de ANSYS-SOLID186 sonuçları için daha yavaștır ve eksenel FD eliptik helis her iki çözüm yöntemi için daha yoğun ağ sıklaştırmasına ihtiyaç duymaktadır. Bunun nedenini, Sekil 7a'daki helis yayı boyunca eliptik helisin eğrilik değerindeki değişiminin hiperboloidal ve fiçı helise göre daha yüksek genliklerde ve daha küçük periyotlarda sürekli değişen salınmalara sahip olması ile açılayabiliriz. Bu yüzden, eliptik helis daha fazla ağ sıklaştırmasına ihtiyaç duymaktadır. Ayrıca, belirtilen helislerin yay boyu değişimlerine ait grafik Şekil 7b'de verilmiştir.
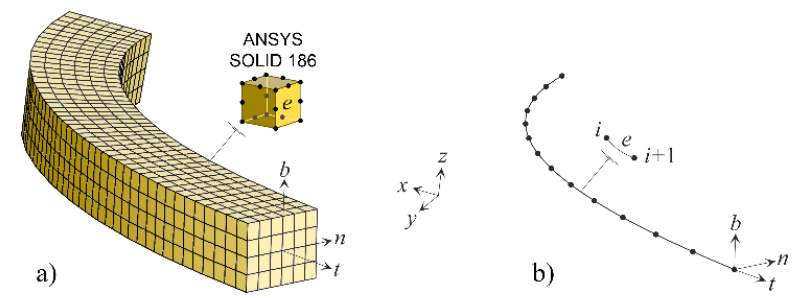

Şekil 6. Frenet koordinat takımında $(t, n, b)$ a) ANSYSSOLID186 elemanı ve b) karışık SE ait eğrisel eleman
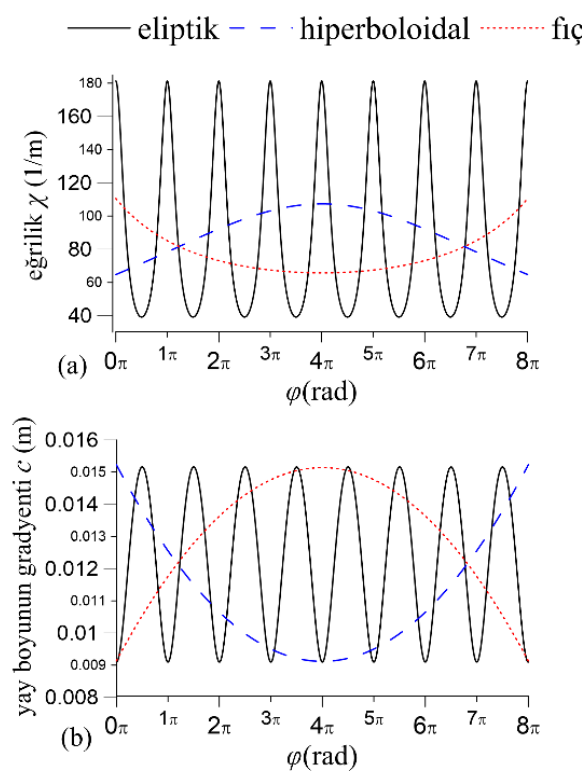

Şekil 7. Eliptik, fiçı ve hiperboloidal helislerin eğrilik ve yay boyu gradyenti fonksiyonlarının yatay açı $0 \leq \varphi \leq 2 \pi n$ aralığında değiş̧imi 


\subsubsection{Parametrik analiz}

Bölüm 3.2.1'de sunulan yakınsama analizi sonuçlarına göre, eksenel FD fıçı ve hiperboloidal helis eliptik helise göre daha az sayıda elman ile yakınsasa da eliptik helis analizinde yeterli yakınsak sonuçları elde etmek için toplamda 400 karışık SE gerekmektedir. Bu nedenle, tüm helis türlerinde yetirince hassas sonuçları sağlayan 400 karışık SE sonuçları kullanılmıştır. Bölüm 3.2 ve 3.2.1 de verilen parametrelere ek olarak problemler ilave sinır koşullarının (ankastre-serbest, serbest ankastre, ankastresabit ve sabit-ankastre) yanı sıra ilave malzeme değişim parametreleri $m=0.5,2,3$ için ele alınmıştır. Eksenel FD fiçı, eliptik ve hiperboloidal helislerin ilk beş frekans değerleri Tablo 3 'te sunulmuştur. Parametrik analizden elde edilen sonuçlara (Tablo 3) göre: en küçük birinci doğal frekans değeri serbest-ankastre sınır koşulu (SK) ve malzeme değişim parametresi $m=1$ değerine sahip eksenel FD fiçı helis için elde edilmiştir. En büyük birinci doğal frekans değeri ise ankastre-ankastre SK ve malzeme değişim parametresi $m=0.5$ değerine sahip eksenel FD hiperboloidal helis için elde edilmiştir. Burada, sınır koşulları sırasıyla helisel çubuğun başlangıç A ve bitiş B noktalarını göstermektedir (Bkz. Şekil 4). Eksenel FD fiçı ve eliptik helislerin birinci doğal frekans değerleri eksenel FD hiperboloidal helis sonuçlarına göre her bir malzeme değişim parametresi $m=0.5,1,2,3$ ve sınır koşulları için $\beta=f_{i}^{m} / f_{\mathrm{h}}^{m}$ ( $i=\mathrm{f}$,e burada $\mathrm{f}$, e ve h sırasıyla fiçı, eliptik ve hiperboloidal helisleri göstermektedir.) formülü üzerinden normalize edilmiştir (Bkz. Şekil 8). Bu oran en büyük değerini $\beta=0.965$ olarak ankastre-ankastre sınır koşulu ve malzeme değişim parametresi $m=3$ sahip eksenel FD eliptik helis için almaktadır. En küçük değerini ise $\beta=0.795$ olarak ankastre-serbest sınır koşulu ve malzeme değişim parametresi $m=0.5$ sahip eksenel FD fiçı helis için almaktadır.

Tablo 3. Eksenel FD dairesel olmayan helislerin ilk beş frekans (Hz) değeri. (400 karışık SE)

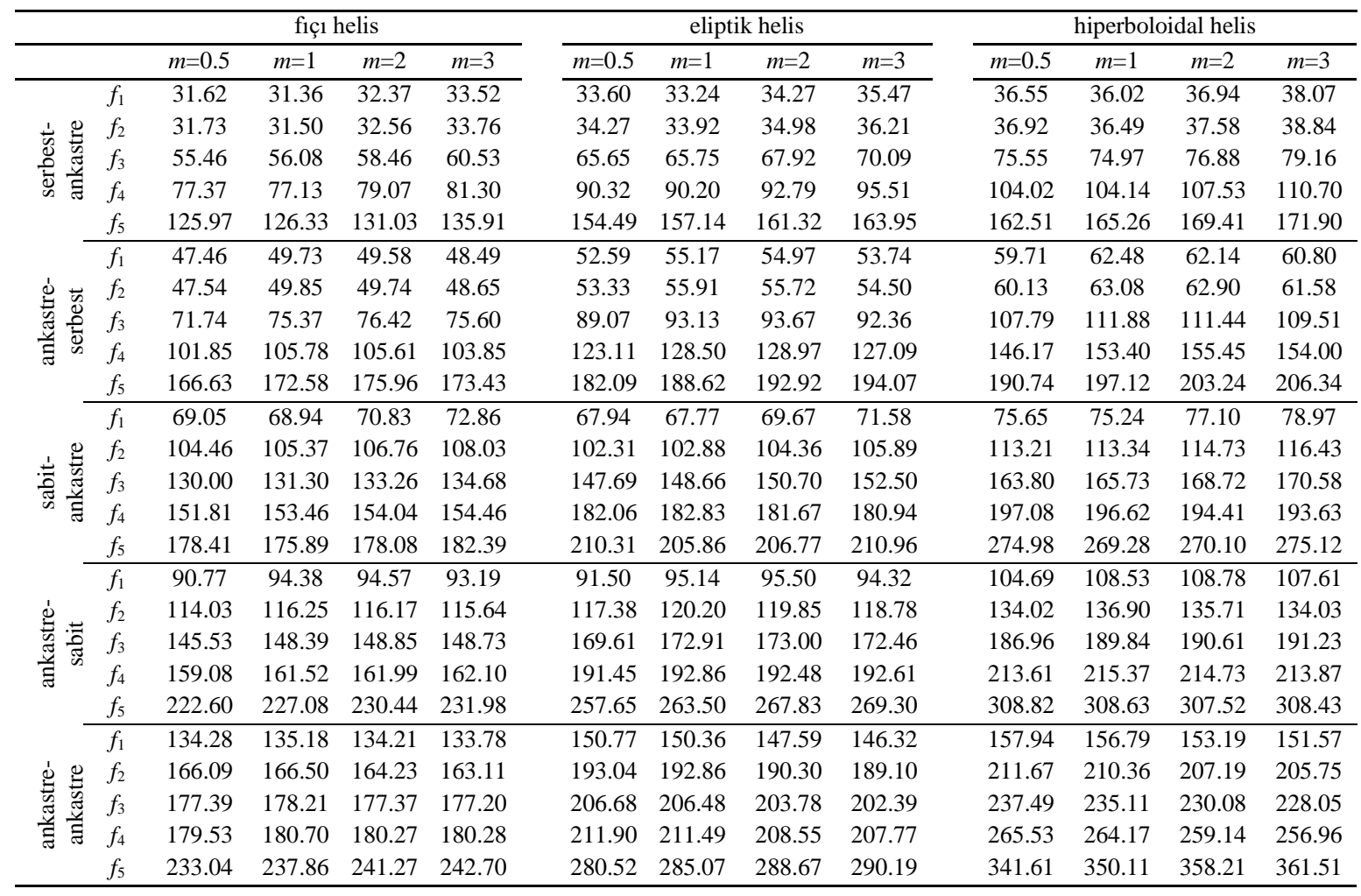

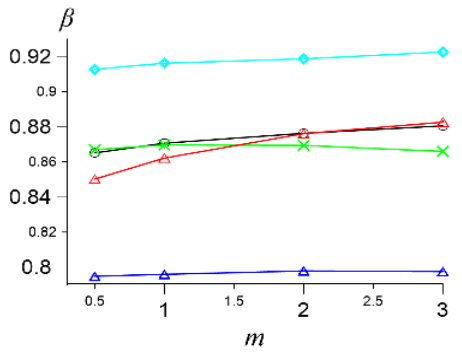

(a)

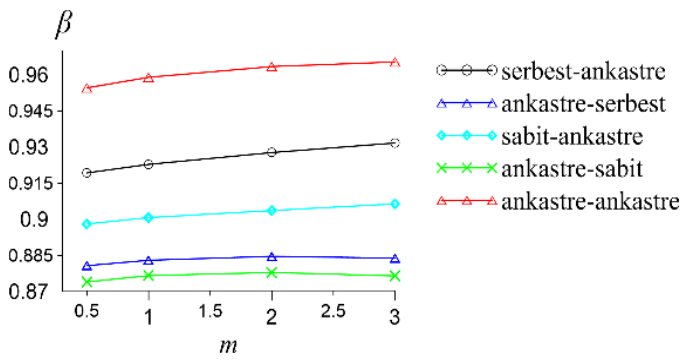

(b)

Şekil. 8. Eksenel FD hiperboloidal helis sonuçlarına göre normalize edilen (a) eksenel FD fiçı helis ve (b) eksenel FD eliptik helis sonuçlarına ait $\beta$ oranın farklı malzeme gradyenti parametresine $(m)$ göre değişimi 


\section{Sonuçlar}

Bu çalışmanın ilk amacı, dairesel olmayan (fiçı, eliptik ve hiperboloidal) Lancret türü helislerin kesin eksen geometrisini tanımlamaktır. Kesin helis geometrisi üstünden yapısal analizler yaklaşık geometri üstünden yapılan analizlere göre hassasiyet anlamında büyük üstünlük sağlamaktadır. Bu değerlendirme hem Ermis ve Omurtag [49] da ve hem de Arıbas vd. [55] de kapsamlı bir biçimde incelenmiștir. $\mathrm{Bu}$ çalıșmanın ikinci amacı, eksenel FD malzemeden imal edilmiş dairesel olmayan (dönel simetrik) kesin geometrili helislerin serbest titreşim analizini karışık SEY ile incelemektir. Bu çalışma kapsamında aşağıdaki sonuçlara varılmıștır

- Literatür ile doğrulama: Eksenel FD doğru eksenli çubukların temel doğal frekansları $[19,22]$ ile kıyaslanmış ve mükemmel bir uyum sağlanmıştır.

- Yakınsama: Dairesel olmayan eksenel FD helisel çubukların yakınsama analizleri, temel doğal frekansları (Bölüm 3.2.1) üzerinden eğrisel karışı sonlu eleman formülasyonu ile ANSYS'in SOLID186 sonlu eleman sonuçları üzerinden gerçekleştirilmiştir. Eğrisel karışık SE formülasyon sonuçları düğüm noktalarındaki toplam bilinmeyen sayıları göz önüne alındığında ANSYS' in SOLID186 eleman sonuçlarına kıyasla daha üstün bir yakınsama performansı göstermektedir (Bkz. Şekil 5).

- Parametrik analiz: Araștırmada seçilen malzeme değișim parametreleri ( $m=0.5,1,2,3)$ göz önüne alındığında sonuçlar göstermiştir ki; serbest-ankastre, sabit-ankastre ve ankastre-ankastre sınır koşulları için, $m$ değeri arttıkça, eksenel FD fiçı ve eliptik helise ait $\beta$ oranları da artmaktadır (Bkz. Şekil 8).

\section{Teşekkür}

Destekleri için Sayın Prof. Dr. Mehmet H. OMURTAG'a sonsuz teșekkürlerimi sunarım.

\section{Çıkar çatışması}

Çıkar çatışması bulunmamaktadır.

\section{Benzerlik oranı (iThenticate): $\% 4$}

\section{Kaynaklar}

[1] Li W and Han B. Research and application of functionally gradient materials. IOP Conf. Series: Materials Science and Engineering 394, 1-7 2018. https://doi.org/ 10.1088/1757899X/394/2/022065.

[2] M. R. Abbas, M. B. Uday, A. M. Noor, N. Ahmad and S. Rajoo, Microstructural evaluation of a slurry based $\mathrm{Ni} / \mathrm{YSZ}$ thermal barrier coating for automotive turbocharger turbine application. Materials \& Design, 109, 47-56, 2016. https://doi.org/10.1016/j.matdes. 2016.07.070

[3] S. R. Dhineshkumar, M. Duraiselvam, S. Natarajan, S. S. Panwar, T. Jena and M. A. Khan Enhancement of strain tolerance of functionally graded LaTi2A19O19 thermal barrier coating through ultra-short pulse based laser texturing. Surface and Coatings Technology, 304,
263-271, 2016. https://doi.org/10.1016/j.surfcoat. 2016.07.018.

[4] S. M. Naga, M. Awaad, H. F. El-Maghraby, A. M. Hassan, M. Elhoriny, A. Killinger and R. Gadow Effect of $\mathrm{La}_{2} \mathrm{Zr}_{2} \mathrm{O}_{7}$ coat on the hot corrosion of multi-layer thermal barrier coatings. Materials \& Design, 102, 1-7, 2016. https://doi.org/10.1016/j.matdes.2016.03.133

[5] K. B. Ghosh, J. Mukhopadhyay and R. N. Basu Functionally graded doped lanthanum cobalt ferrite and ceria-based composite interlayers for advancing the performance stability in solid oxide fuel cell. Journal of Power Sources, 328, 15-27, 2016. https://doi.org/10. 1016/j.jpowsour.2016.07.106

[6] A. Solla, D. Bellucci and V. Cannillo, Functionally graded materials for orthopedic applications - an update on design and manufacturing. Biotechnology Advances 34, 504-531, 2016. https://doi.org/10.1016/ j.biotechadv.2015.12.013

[7] J. Cherusseri, R. Scharma and K. K. Kar Helically coiled carbon nanotube electrodes for flexible supercapacitors. Carbon, 105, 113-125, 2016. https://doi.org/10.1016/j.carbon.2016.04.019

[8] E. H. Egelman Three-dimensional reconstruction of helical polymers. Archives of Biochemistry and Biophysics, 581, 54-58, 2015. https://doi.org/10.1016 /j.abb.2015.04.004

[9] X Jian, D. Wang, H. Liu, M. Jiang, Z. Zhou, J. Lu, X. $\mathrm{Xu}$, Y. Wang, L. Wang, Z. Gong, M. Yang, J. Gou and D. Hui, Controllable synthesis of carbon coils and growth mechanism for twinning double-helix catalyzed by Ni nanoparticle. Composites Part B: Engineering, 61, 350-357, 2014. https://doi.org/10.1016/ j.compositesb.2013.06.010

[10] A. Baratta and I. Corbi, Equilibrium models for helicoidal laterally supported staircases. Computers \& Structures,124, 21-28, 2013. https://doi.org/10.1016/ j.compstruc.2012.11.007

[11] M. Angelillo, Static analysis of a Guastavino helical stair as a layered masonry shell. Composite Structures $119,298-304, \quad 2015 . \quad$ https://doi.org/10.1016/ j.compstruct.2014.09.007

[12] N. Gao and Y. Zhang, A low frequency underwater metastructure composed by helix metal and viscoelastic damping rubber. Journal of Vibration and Control, 25(3), 538-548, 2018. https://doi.org/10.1177/ 1077546318788446

[13] N. Barbieri, R. Barbieri, R. A. da Silva, M. J. Mannala and L.S.A.V. Barbieri, Nonlinear dynamic analysis of wire-rope isolator and Stockbridge damper. Nonlinear Dynamics 86, 501-512, 2016. https://doi.org/10.1007/ s11071-016-2903-1

[14] L. Wu, Q.-s. Wang and I. Elishakoff, Semi-inverse method for axially functionally graded beams with an anti-symmetric vibration mode. Journal of Sound and Vibration, 284, 1190-1202, 2005. https://doi.org/10. 1016/j.jsv.2004.08.038

[15] M. Aydogdu, Semi-inverse method for vibration and buckling of axially functionally graded beams. Journal 
of Reinforced Plastics and Composites, 27, 683-691 2008. https://doi.org/10.1177/0731684407081369

[16] Shahba, R. Attarnejad, M. T. Marvi and S. Hajilar, Free vibration and stability analysis of axially functionally graded tapered Timoshenko beams with classical and non-classical boundary conditions. Composites Part B: Engineering 42, 801-808, 2011. https://doi.org/10. 1016/j.compositesb.2011.01.017.

[17] Y. Huang, L.-E. Yang and Q.-Z. Luo, Free vibration of axially functionally graded Timoshenko beams with non-uniform cross-section. Composites Part B: Engineering 45, 1493-1498, 2013. https://doi.org/10. 1016/j.compositesb.2012.09.015

[18] X.-F. Li, Y.-A. Kang and J.-X. Wu, Exact frequency equations of free vibration of exponentially functionally graded beams. Applied Acoustics, 74, 413-420, 2013. https://doi.org/10.1016/j.apacoust.20 12.08 .003

[19] S. Rajasekaran and E. N. Tochaei, Free vibration analysis of axially functionally graded tapered Timoshenko beams using differential transformation element method and differential quadrature element method of lowest-order. Meccanica 49(4), 995-1009, 2014. https: //doi.org/10.1007/s11012-013-9847-z

[20] K. Sarkar and R. Ganguli, Closed-form solutions for axially functionally graded Timoshenko beams having uniform cross-section and fixed-fixed boundary condition. Composites Part B: Engineering, 58, 361370, 2014. https://doi.org/10.1016/j.compositesb.20 13.10.077

[21] F. F. Calim, Transient analysis of axially functionally graded Timoshenko beams with variable cross-section. Composites Part B: Engineering 98, 472-483, 2016. https://doi.org/10.1016/j.compositesb.2016.05.040

[22] Y. Zhao, Y. Huang and M. Guo, A novel approach for free vibration of axially functionally graded beams with non-uniform cross-section based on Chebyshev polynomials theory. Composite Structures, 168, 277 284, 2017. https://doi.org/10.1016/j.compstruct.2017. 02.012

[23] D. Cao, Y. Gao, M. Yao and W. Zhang, Free vibration of axially functionally graded beams using the asymptotic development method. Engineering Structures, 173, 442-448, 2018. https://doi.org/ 10.1016/j.engstruct.20 18.06.111

[24] S. Šalinic, A. Obradovic and A. Tomovic, Free vibration analysis of axially functionally graded tapered, stepped, and continuously segmented rods and beams. Composites Part B: Engineering, 150, 135-143, $2018 . \quad$ https://doi.org/10.1016/j.compositesb. 2018.05.060

[25] X. Li, L. Li, Y. Hu, Z. Ding and W. Deng, Bending, buckling and vibration of axially functionally graded beams based on nonlocal strain gradient theory. Composite Structures 165, 250-265, 2017. https://doi.org/10.1016/j.compstruct.2017.01.032

[26] M. Sari, M. Shaat and A. Abdelkefi, Frequency and mode veering phenomena of axially functionally graded non-uniform beams with nonlocal residuals.
Composite Structures, 163, 280-292, 2017. https://doi.org/10.10 16/j.compstruct.2016.11.093

[27] M. H. Ghayesh and H. Farokhi, Mechanics of tapered axially functionally graded shallow arches. Composite Structures 188, 233-241, 2018. https://doi.org/10. 1016/ j.compstruct.2017.11.017

[28] M. Chen, G. Jin, Y. Zhang, F. Niu and Z. Liu, Threedimensional vibration analysis of beams with axial functionally graded materials and variable thickness. Composite Structures, 207, 304-322, 2019. https://doi.org/10.1016/j.compstruct.2018.09.029

[29] X. Zhang, Z. Ye and Y. Zhou, A Jacobi polynomial based approximation for free vibration analysis of axially functionally graded material beams. Composite Structures, 225, 111070, 2019. https://doi.org/10.1016/ j.compstruct.2019.111070

[30] R. Talebitooti, S. O. Rezazadeh and A. Amiri, Comprehensive semi-analytical vibration analysis of rotating tapered AFG nanobeams based on nonlocal elasticity theory considering various boundary conditions via differential transformation method. Composites Part B: Engineering, 160, 412-435, 2019. https://doi.org/10.1016/j.compositesb.2018.12.085

[31] S. Rajasekaran, Analysis of curved beams using a new differential transformation based curved beam element. Meccanica, 49, 863-886, 2014. https://doi.org/10.1007 /s11012-013-9835-3

[32] G. C. Tsiatas and A. E. Charalampakis, Optimizing the natural frequencies of axially functionally graded beams and arches. Composite Structures, 160, 256266, 2017. https://doi.org/10.1016/j.compstruct. 2016.10 .057

[33] J. K. Lee and B. K. Lee, In-plane free vibration of uniform circular arches made of axially functionally graded materials. International Journal of Structural Stability and Dynamics, 19, 1950084, 2019. https://doi.org/10. 1142/S0219455419500846

[34] R. Noori, T. A. Aslan and B. Temel, An efficient approach for in-plane free and forced vibrations of axially functionally graded parabolic arches with nonuniform cross section. Composite Structures, 200, 701-710, 2018. https://doi.org/10.1016/j.compstruct. 2018.05.07 7

[35] B. Temel and A. R. Noori, Out-of-plane vibrations of shear-deformable AFG cycloidal beams with variable cross section. Applied Acoustics, 155, 84-96, 2019. https://doi.org/10.1016/j.apacoust.2019.05.010

[36] F.F. Calim and Y.C. Cuma, Vibration analysis of nonuniform hyperboloidal and barrel helices made of functionally graded material. Mechanics Based Design of Structures and Machines, 2020. https://doi.org/ 10.1080/15397734.2020.1822181

[37] K. Nagaya, S. Takeda and Y. Nakata, Free vibration of coil springs of arbitrary shape. International Journal for Numerical Methods in Engineering 23, 1081-1099, 1986. https://doi.org/10.1002/nme.1620230607

[38] V. Y1ldirım, A parametric study on the free vibration of noncylindrical helical springs. Journal of Applied 
Mechanics, 65(1), 157-163, 1998. https://doi.org/10. 1115/1.2789019

[39] W. Busool and M. Eisenberger, Free vibration of helicoidal beams of arbitrary shape and variable cross section. Journal of Vibration and Acoustic, 124, 397 409, 2002. https://doi.org/10.1115/1.1468870

[40] V. Yıldirım, A parametric study on the natural frequencies of unidirectional composite conical springs. Communications in Numerical Methods in Engineering, 20, 207-227, 2004. https://doi.org/10.10 02/cnm.661

[41] J. Lee, Free vibration analysis of non-cylindrical helical springs by the pseudospectral method. Journal of Sound and Vibration 305, 543-551, 2007. https://doi.org/10.1016/j.jsv.2006.11.008

[42] A. Yu and Y. Hao, Effect of warping on natural frequencies of symmetrical cross-ply laminated composite non-cylindrical helical springs. International Journal of Mechanical Sciences, 74, 65-72 (2013). https://doi.org/10.1016/j.ijmecsci.2013.04.010

[43] N. Eratli, M. Ermis, M. H. Omurtag, Free vibration analysis of helicoidal bars with thin-walled circular tube cross-section via mixed finite element method. Sigma Journal of Engineering and Natural Sciences, 33, 200-218, 2015.

[44] N. Eratli, M. Yilmaz, K. Darilmaz and M.H. Omurtag, Dynamic analysis of helicoidal bars with non-circular cross-sections via mixed FEM. Structural Engineering and Mechanics, 57, 221-238, 2016. https://doi.org/10. 12989/sem.2016.57.2.221.

[45] F. F. Çalım, Dynamic analysis of composite coil springs of arbitrary shape. Composites Part B: Engineering, 40, 741-757, 2009. https://doi.org/ 10.10 16/j.compositesb.2009.04.017.

[46] F. F. Çalım, Forced vibration of helical rods of arbitrary shape. Mechanics Research Communications, 36, 882891, 2009. https://doi.org/10.1016/j.mechrescom.2009 .07 .007 .

[47] A. Yampolsky and A. Opariy, Generalized helices in three-dimensional Lie groups. Turkish Journal of Mathematics, 43, 1447-1455, 2019.

[48] Ü. Çiftçi, A generalization of Lancret's theorem, J. Geom. Phys. 59, 1597-1603, 2009. https://doi.org/10. 1016/j.geomphys.2009.07.016.

[49] M. Ermis and M.H. Omurtag, Static and dynamic analysis of conical helices based on exact geometry via mixed FEM. International Journal of Mechanical Sciences, 131, 296-304, 2017. https://doi.org/10. 1016/j.ijmecsci.2017.07.010.

[50] M. Ermis, Static and dynamic analysis of non-circular helical bars based on exact geometry. Ph. D Thesis, Istanbul Technical University, İstanbul, Türkiye 2019.

[51] C.S. Jog, I.S. Mokashi, A finite element method for the Saint-Venant torsion and bending problems for prismatic beams. Computers \& Structures, 135, 62-72 2014. https://doi.org/10.1016/j.compstruc.2014.01.0 10
[52] R. Barretta, L. Feo and R. Luciano, Some closed-form solutions of functionally graded beams undergoing nonuniform torsion. Composite Structures, 123, 132136, 2015. https://doi.org/10.1016/j.compstruct.2014. 12.027

[53] K. Darılmaz, E. Orakdögen and K. Girgin, SaintVenant torsion of arbitrarily shaped orthotropic composite or FGM sections by a hybrid finite element approach. Acta Mechanica, 229, 1387-1398, 2018. https://doi.org/10.1007/s00707-017-2067-1

[54] U. N. Aribas Kompozit eğrisel kirişlerde kesit çarpilmasi gözetilerek normal ve kayma gerilmelerinin karışık sonlu elemanlarla analizi. Niğde Ömer Halisdemir Üniversitesi Mühendislik Bilimleri Dergisi, 8(3), 16-29, 2019. https://doi.org/10.28948/ngumuh. 619591

[55] U. N. Aribas, M. Ermis, N. Eratli and M. H. Omurtag, The static and dynamic analyses of warping included composite exact conical helix by mixed FEM. Composites Part B: Engineering, 160, 285-297, 2019. https://doi.org/10.1016/j.compositesb.2018.10.018

[56] U. N. Aribas, M. Ermis, A. Kutlu, N. Eratli and M. H. Omurtag, Forced vibration analysis of compositegeometrically exact elliptical cone helices via mixed FEM. Mechanics of Advanced Materials and Structures, 1-19, 2020. https://doi.org/10.1080/ 15376494.2020.1824048.

[57] H.C. Tsai and J. M. Kelly, Buckling of short beams with warping effect included, International Journal of Solids and Structures. 42(1), 239-253, 2005. https://doi.org/10.1016/j.ijsolstr.2004.07.021

[58] Genoese, A. Genoese, A. Bilotta and G. Garcea, A geometrically exact beam model with non-uniform warping coherently derived from the Saint Venant rod. Engineering Structures 68, 33-46, 2014. https://doi.org/10.1016/j.engstruct.2014.02.024

[59] K. Yoon, P. S. Lee and D. N. Kim, Geometrically nonlinear finite element analysis of functionally graded 3D beams considering warping effects. Composite Structures 132, 1231-1247, 2015. https://doi.org/ 10.1016/j.compstruct.2015.07.024

[60] Di Egidio, A. Contento and F. Vestroni, The role of nonlinear torsional contributions on the stability of flexural-torsional oscillations of open-cross section beams. Journal of Sound and Vibration 358, 236-250 2015. https://doi.org/10.1016/j.jsv.2015.08.004

[61] ANSYS $®$, Academic Research Mechanical, Release 17.1, Canonsburg, Pennsylvania, 2016.

[62] M. H. Omurtag and A. Y. Aköz, The mixed finite element solution of helical beams with variable crosssection under arbitrary loading.. Computers \& Structures, 43, 325-331, 1992. https://doi.org/10.1016/ 0045-7949 (92)90149-T.

[63] J.T. Oden and J.N. Reddy, An introduction to the mathematical theory of finite elements, John Wiley \& Sons, Limited, 1976. 